\title{
Hand-Sewn Running Barbed Suture versus Endoscopic Stapler Closure of the Mesenteric Defects in Retrocolic and Retrogastric RYGB: A Comparative Case-Matched Study
}

\author{
V. Simonelli*, G. Orlando, A. Zolotas, C. Arendt, L. Arru, V. Poulain, J. S. Azagra, M. Goergen \\ Centre Hospitalier de Luxembour, Luxembourg Ville, Luxembourg \\ Email: vincyg@hotmail.it
}

Received 22 June 2015; accepted 26 July 2015; published 29 July 2015

Copyright $(\odot 2015$ by authors and Scientific Research Publishing Inc.

This work is licensed under the Creative Commons Attribution International License (CC BY).

http://creativecommons.org/licenses/by/4.0/

(c) (i) Open Access

\section{Abstract}

Background: Obesity is nowadays a major health concern in Western countries and the number of bariatric surgical procedures being performed worldwide is vertiginously rising. The Laparoscopic Roux-en-Y gastric bypass (LRYGB) has become the gold standard bariatric procedure. The retrocolic retrogastric approach is closer to normal anatomy and it is associated to a lower rate of anastomotic leak, a lower rate of gastrojejunal stricture and a lower rate of marginal ulcers; therefore the problem of small bowel obstruction due to internal hernia (IH) has to be faced. The meticulous closure of all possible mesenteric defects with running, non-absorbable sutures may reduce the rate of this complication, but it can be challenging for the surgeon and it rises the operating time (OT). This study has conducted in a context of optimization of our "Fast Track-type" recovery protocol and it aims to compare the rate of early IH and the OT difference when mesenteric defects are closed using running non-absorbable barbed suture or an endoscopic stapler. Materials and Methods: From December 2014 to February 2015 a single-surgeon consecutive series of 22 patients undergoing retrocolic restrogastric LRYBP in our high volume obesity centre has been retrospectively extrapolated from our prospective longitudinal database. We recorded the overall $O T$ and relative rate of $I H$ in patients who received a $15-\mathrm{cm}$ non-absorbable $V-L^{-C^{T M} 1}$ (group A, 11 patients) or Endopath ${ }^{\text {TM }}$ EMS, endoscopic multifeed stapler ${ }^{2}$ (group B, 11 patients) defect. Results: The mean OT was 77.36 minutes in the group $A$ and 60.90 minutes in the group $B(P$ value 0.066 ). 0 patients $(0 \%)$ in the group A versus 4 patients $(36.3 \%)$ in the group B developed IH within 30

\footnotetext{
Corresponding author.

${ }^{1}$ Covidien, Mansfield, MA, USA.

${ }^{2}$ Ethicon Endo-Surgery, LLC, Guaynabo, Puerto Rico, USA.
}

How to cite this paper: Simonelli, V., et al. (2015) Hand-Sewn Running Barbed Suture versus Endoscopic Stapler Closure of the Mesenteric Defects in Retrocolic and Retrogastric RYGB: A Comparative Case-Matched Study. Surgical Science, 6, 346351. http://dx.doi.org/10.4236/ss.2015.68051 
days (two-tailed P value at chi-square test: 0.02 ). Conclusion: Early rate of small bowel obstruction due to IH is extremely higher with the use of an endoscopic stapler instead of non-absorbable barber suture and there is not significant difference in the OT; more prospective randomized trials observing bigger series of patients with longer follow-up are needed to validate our study.

\section{Keywords}

\section{Laparoscopy, Gastric Bypass, Fastrack, Internal Hernia}

\section{Introduction}

Obesity has become a major health concern in Western countries. This has resulted in a steady rise in the number of bariatric surgical procedures being performed worldwide. Of these, the Laparoscopic Roux-en-Y gastric bypass (LRYGB) has become the gold standard bariatric procedure to which all the others are compared [1] and accounted for about 45\% of all bariatric surgeries performed worldwide in 2013, whereas in 2015 IFSO data show the rise in prevalence of Sleeve Gastrectomy [2].

LRYGB can be accomplished using either an antecolic or retrocolic approach.

Many surgical teams prefer the antecolic LRYGB because it is easier to perform with a reduction of operating time (OT) and hospital stay [3], as many others prefer better the retrocolic, retrogastric approach because it is closer to anatomical structure; it is associated to a lower rate of anastomotic leaks [4], a significant lower rate of gastrojejunal strictures [5] and a significant lower rate of marginal ulcers [6]; therefore the retrocolic approach seems to be a risk factor for intestinal obstruction and internal hernia (IH) compared to the antecolic one when the mesenteric defects are not well closed [5] [7].

The onset of an IH can be a severe complication (1\% to 9\%) and produce catastrophic consequences if not diagnosed and treated on time. To reduce the rate of this complication in the retrocolic, retrogastric LYRGB it's mandatory to close meticulously all possible defects with running, non-absorbable sutures [8] and that is possible but more challenging for the surgeon and also it needs longer OT.

This study has been conducted in a context of optimization of our "Fast-Track" type recovery protocol and it aims to compare the incidence of short-term IH and the OT difference when mesenteric defects are closed using hand-sewn running non-absorbable suture or an endoscopic stapler.

\section{Materials and Methods}

A retrospective review of our prospectively collected database has been conducted in our high volume obesity centre, “Centre Hospitalier de Luxembourg”, Luxembourg, from December 2014 to February 2015.

Twenty-two consecutive single-surgeon obesity patients undergoing retrocolic restrogastric LRYBP have been entered in our comparative case-matched study.

All the patients considered participated in a "Fast Track-type" recovery protocol.

We recorded all patients that received an hand-sewn running suture (group A) or automatic mechanical endostapling device (group B).

\section{Surgical Technique}

All the patients included underwent 5 trocarts retrocolic retrogastric LRYBP; they all have been operated from the same surgeon.

The first time of the operation, with the surgeon on right side of the patient, consist in the creation of a passage throw the trasverse mesocolon in order to get the posterior gastric wall from the infra-mesocolic space. A biliary limb is stapled at $50 \mathrm{~cm}$ from the Treitz ligament; the meso between the resulting two limbs is not divided so that the Petersen defect is virtual in our technic and doesn't need to be closed. Then $120 \mathrm{~cm}$ are measured from the staple line of the alimentary side and a side-to-side jejunojejunostomy is here performed full-mechanically.

In the group A the jejunojejunostomy site (JJ) mesenteric defect closure is performed with a 3-0, 15-cm-long, 
non-adsorbableV-Loc ${ }^{\mathrm{TM}}{ }^{1}$.

In the group B the JJ mesenteric defect closure is performed, with a single stapling line employing the endoscopic multifeed stapler Endopath ${ }^{\mathrm{TM}} \mathrm{EMS}^{2}$.

The alimentary limb is then leaded throw the mesocolic passage into the epiploic retro-cavity and the trasmesocolic (TM) defect is closed with a 3-0, 15-cm-long, non-absorbable V-Loc ${ }^{\mathrm{TM}}$ in the group A and with a single stapling line employing the endoscopic multifeed stapler Endopath ${ }^{\mathrm{TM}}$ EMSin the group B.

The second time of the operation, with the surgeon in front of the patient set-up in French position, consist in the creation of a gastric pouch of $10 \mathrm{ml}$; then the alimentary limb is stapled with the gastric pouch side-to-side and the gastrojejunostomy is finally completed using a 3-0 absorbable V-Loc ${ }^{\mathrm{TM}}$.

The overall OT in both the groups has been compared and the incidence of early small bowel occlusion for IH within 30 days from the operation has been then evaluated.

\section{Results}

Between December 2014 and February 2015 twenty-two single-surgeon consecutives obesity patients underwent a LRYBP in our obesity centre.

No selection criteria have been applied but patients with bad controlled diabetes, chronic hearth failure and Gold III COPD or higher and all the redo surgeries have been excluded.

The group A is composed of eleven patients ( 9 women and 2 men) with a mean age of 44.00 years (range, 19 - 63 years) and a mean BMI of 46.32 (range, $34-58.2 \mathrm{Kg} / \mathrm{m}^{2}$ ); the group B is composed of eleven patients (9 women and 2 men) with a mean age of 46.63 (range, 33 - 59 years) and a mean BMI of 44.20 (range, 35.4 - 52 $\mathrm{Kg} / \mathrm{m}^{2}$ ) (Table 1).

There was no perioperative death or complication.

The mean OT from incision to closure was 77.36 minutes (range, 45 - 100 minutes) in the group A and 60.90 minutes (range, 45 - 90 minutes) in the group B $(\mathrm{p}=0.066)$ (Table 2).

0 patients (0\%) in the group A versus 4 patients (36.3\%) in the group B developed IH within 30 days (twotailed $\mathrm{P}$ value at chi-square test: 0.02 ) (Table 2, Figure 1 ).

\section{Discussion}

Morbid obesity is a growing epidemic in the Western world that adversely affects the quality of life and life expectancy [9]-[11]. For that reason the number of bariatric surgical procedures is extremely rising worldwide and the LRYGB is generally considered the gold-standard operation [12]. In high volume obesity centres the choice of the good surgical technique is important to improve outcomes, to reduce the overall OT and to avoid perioperative complications, so that also the recovery costs can be reduced.

In our obesity surgical team we strongly believe that the retrocolic retrogastric approach is associated with a lower rate of anastomotic leak, a lower gastrojejunal stricture rate and a lower rate of marginal ulcers; therefore

Table 1. Main characteristics of the patients in the two groups. (n): number of patients.

\begin{tabular}{cccccc}
\hline & (n) & Women & Men & Age, mean & BMI, mean \\
Group A & 11 & 9 & 2 & $44.00(19-63)$ & $46.32(34-58.2)$ \\
Group B & 11 & 9 & 2 & $46.63(33-59)$ & $44.20(35.4-52)$ \\
\hline
\end{tabular}

Table 2. Comparison between operative time in minutes and internal hernia rate in the two groups. Mean OT P value $=$ 0.066 (Student's test not significant). IH rate two-tailed P value $=0.02$ (chi-square test significant). OT: operating time. SD: standard deviation. IH: internal hernia.

\begin{tabular}{cccc}
\hline & Group A & Group B & P values \\
\hline OT, mean & $77.36(45-100)$ & $60.90(45-90)$ & $>0.05$ (not significant) \\
SD & 19.15 & 15.55 & $<0.05$ (significant) \\
IH rate & $0 \%$ & $36.3 \%$ & $<$ \\
\hline
\end{tabular}




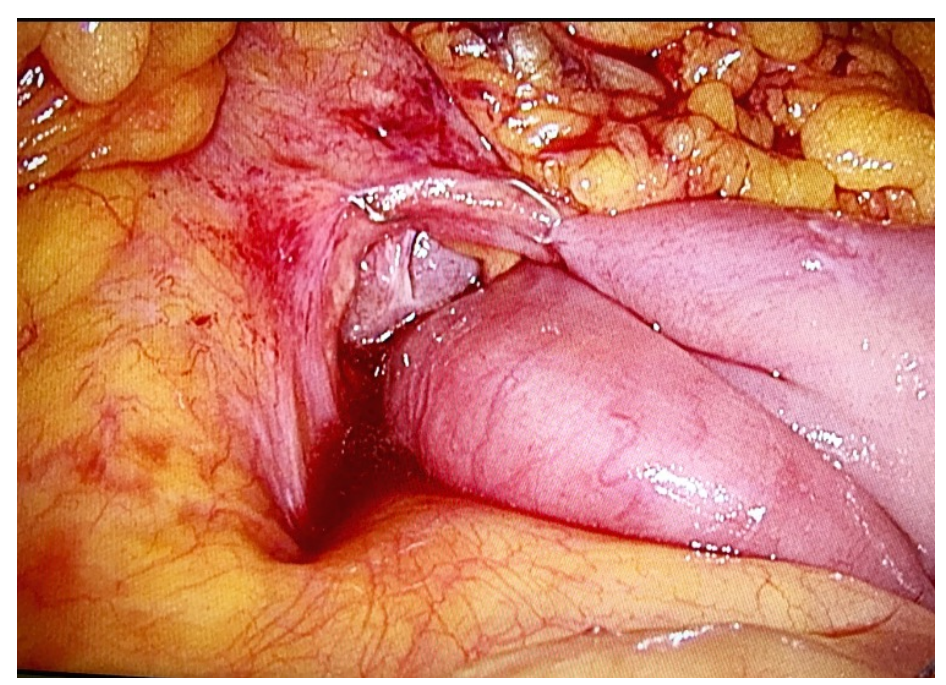

Figure 1. Per-operative picture showing the opening in the transverse mesocolon after reduction of an internal hernia in a patient where the defect had been closed with staples.

the problem of small bowel obstruction due to IH has to be faced with this approach [13]. Various groups advocate the meticulous closure of all possible mesenteric defects with running, non-absorbable sutures may reduce the rate of this complication [8] [13] [14].

To close the mesenteric defects is feasible with several materials and techniques, as separated running (traditional or barbed) non-absorbable stitches or an endoscopic stapler, but the choice can influence the OT.

"Time is money" and in Luxembourg 1 minute use of the operation room coasts 16 euros, so reducing the overall OT without rise the rate of complications is very important.

In our study the mean OT is relatively short and not significantly different in the 2 groups observed.

In our technique we don't split the mesentery of biliary and alimentary limbs, so the Petersen defect is virtual and it doesn't need to be closed.

The advantages of using non-absorbable barbed sutures are well known and they are the following: they are safe and reproducible with a short learning curve [15]; assistance is not required to maintain suture tension; the suture does not slip [16]; it shortens the total OT [17] and it seems to have similar 30-day outcomes when compared with traditional suture [18].

The endoscopic stapler is really easy and fast to use for the closure of the mesentery, the only difficulty that we experienced it has been the fact that the two branches of the staple are not well visible with a $0^{\circ}$ optic, so the device has for that a lack of precision.

Only short-term results within 30 days have been studied in our study, the long-terms results for both of the closure methods are still unknown.

Our series is short but we have been obliged to interrupt the study because the IH rate was already too high and not acceptable for us in the group B with the first eleven patients (Figure 1).

Maybe closing the mesenteric defects with 2 or more line of staples could have reduced the IH rate, but anyway there is any significant difference in the mean OT in the 2 groups.

\section{Limitations}

The main limitation of our study is the retrospective design. However this may represent a starting point for the implementation of randomized controlled trials on larger samples in order to assess which approach is associated with better long-term outcomes with regards to mesenteric defects closure. Moreover a longer follow-up is needed in order to assess long-term outcomes in this setting.

\section{Conclusion}

The rate of small bowel obstruction due to IH is extremely higher with the use of an endoscopic stapler instead 
of non-absorbable barbed suture and there is not significant difference in the relative mean OT; however more prospective randomized trials observing bigger series of patients with longer follow-up are needed to validate our study.

\section{Disclosure Statement}

Simonelli Vincenzo, Orlando Gennaro, Zolotas Aias, Arendt Charlie, Arru Luca, Poulain Virginie, Azagra Juan Santiago, Goergen Martine have no conflict of interest to diclare.

\section{References}

[1] Nguyen, N.T., DeMaria, E., Ikramuddin, S., et al. (2008) The SAGES Manual: A Practical Guide to Bariatric Surgery. Springer, New York. http://dx.doi.org/10.1007/978-0-387-69171-8

[2] Angrisani, L., Santonicola, A., Formisano, G., Buchwald, H. and Scopinaro, N. (2015) Bariatric Surgery Worldwide 2013. Obesity Surgery.

[3] Müller, M.K., Guber, J., Wildi, S., Guber, I., Clavien, P.A. and Weber, M. (2007) Three-Year Follow-Up Study of Retrocolic versus Antecolic Laparoscopic Roux-en-Y Gastric Bypass. Obesity Surgery, 17, 889-893. http://dx.doi.org/10.1007/s11695-007-9165-4

[4] Edwards, M.A., Jones, D.B., Ellsmere, J., Grinbaum, R. and Schneider, B.E. (2007) Anastomotic Leak Following Antecolic versus Retrocolic Laparoscopic Roux-en-Y Gastric Bypass for Morbid Obesity. Obesity Surgery, 17, $292-297$. http://dx.doi.org/10.1007/s11695-007-9048-8

[5] Ribeiro-Parenti, L., Arapis, K., Chosidow, D., Dumont, J.L., Demetriou, M. and Marmuse, J.P. (2015) Gastrojejunostomy Stricture Rate: Comparison between Antecolic and Retrocolic Laparoscopic Roux-en-Y Gastric Bypass. Surgery for Obesity and Related Diseases.

[6] Ribeiro-Parenti, L., Arapis, K., Chosidow, D. and Marmuse, J.P. (2015) Comparison of Marginal Ulcer Rates between Antecolic and Retrocolic Laparoscopic Roux-en-Y Gastric Bypass. Obesity Surgery, 25, 215-221. http://dx.doi.org/10.1007/s11695-014-1392-X

[7] Escalona, A., Devaud, N., Perez, G., Crovari, F., Boza, C., Viviani, P., Ibanez, L. and Guzman, S. (2007) Antecolic versus Retrocolic Alimentary Limb in Laparoscopic Roux-en-Y Gastric Bypass: A Comparative Study. Surgery for Obesity and Related Diseases, 3, 423-427. http://dx.doi.org/10.1016/j.soard.2007.04.005

[8] Higa, H.D., Ho, T. and Boone, K.B. (2003) Internal Hernias after Laparoscopic Roux-en-Y Gastric Bypass: Incidence, Treatment and Prevention. Obesity Surgery, 13, 350-354. http://dx.doi.org/10.1381/096089203765887642

[9] Buchwald, H. and Oien, D.M. (2009) Metabolic/Bariatric Surgery Worldwide 2008. Obesity Surgery, 19, $1605-1611$. http://dx.doi.org/10.1007/s11695-009-0014-5

[10] McCarty, T.M., Arnold, D.T., Lamont, J.P., Fisher, T.L. and Kuhn, J.A. (2005) Optimizing Outcomes in Bariatric Surgery: Outpatient Laparoscopic Gastric Bypass. Annals of Surgery, 242, 494-498. http://dx.doi.org/10.1097/01.sla.0000183354.66073.4c

[11] Fares, L.G., Reeder, R.C., Bock, J. and Batezel, V. (2008) 23-Hour Stay Outcomes for Laparoscopic Roux-en-Y Gastric Bypass in a Small, Teaching Community Hospital. American Surgeon, 74, 1206-1210.

[12] Thomas, H. and Agrawal, S. (2011) Systematic Review of 23-Hour (Outpatient) Stay Laparoscopic Gastric Bypass Surgery. Journal of Laparoendoscopic and Advanced Surgical Techniques. Part A, 21, 677-681. http://dx.doi.org/10.1089/lap.2011.0042

[13] Steele, K.E., Prokopowicz, G.P., Magnuson, T., et al. (2008) Laparoscopic Antecolic Roux-en-Y Gastric Bypass with Closure of Internal Defects Leads to Fewer Internal Hernias than the Retrocolic Approach. Surgical Endoscopy, 22, 2056-2061. http://dx.doi.org/10.1007/s00464-008-9749-7

[14] Carmody, B., De Maria, E.J., Jamal, M., et al. (2005) Internal Hernia after Laparoscopic Roux-en-Y Gastric Bypass. Surgery for Obesity and Related Diseases, 1, 543-548. http://dx.doi.org/10.1016/j.soard.2005.08.005

[15] Facy, O., De Blasi, V., Goergen, M., Arru, L., De Magistris, L. and Azagra, J.S. (2013) Laparoscopic Gastrointestinal Anastomoses Using Knotless Barbed Sutures Are Safe and Reproducible: A Single-Center Experience with 201 Patients. Surgical Endoscopy, 27, 3841-3845. http://dx.doi.org/10.1007/s00464-013-2992-6

[16] Murtha, A.P., Kaplan, A.L., Paglia, M.J., et al. (2006) Evaluation of a Novel Technique for Wound Closure Using a Barbed Suture. Plastic and Reconstructive Surgery, 117, 1769-1780. http://dx.doi.org/10.1097/01.prs.0000209971.08264.b0

[17] Costantino, F., Dente, M., Perrin, P., et al. (2013) Barbed Unidirectional V-Loc 180 Suture in Laparoscopic Roux-en-Y Gastric Bypass: A Study Comparing Unidirectional Barbed Monofilament and Multifilament Absorbable Suture. Sur- 
gical Endoscopy, 27, 3846-3851. http://dx.doi.org/10.1007/s00464-013-2993-5

[18] Tyner, R.P., Clifton, G.T. and Fenton, S.J. (2013) Hande-Sewn Gastrojejunostomy Using Knotless Unidirectional Barbed Absorbable Suture during Laparoscopi Gastric Bypass. Surgical Endoscopy, 27, 1360-1366. http://dx.doi.org/10.1007/s00464-012-2616-6 